\title{
Severe aortic stenosis has blunted myocardial T1 relaxation response to vasodilator stress: a cardiac magnetic resonance adenosine stress test study
}

\author{
Masliza Mahmod ${ }^{1 *}$, Stefan K Piechnik ${ }^{1}$, Eylem Levelt ${ }^{1}$, Vanessa M Ferreira ${ }^{1}$, Jane M Francis ${ }^{1}$, Andrew Lewis $^{1}$, \\ Nikhil Pal', Houman Ashrafian', Stefan Neubauer', Theodoros D Karamitsos ${ }^{1,2}$
}

From 18th Annual SCMR Scientific Sessions

Nice, France. 4-7 February 2015

\section{Background}

Aortic stenosis is characterized by impaired myocardial perfusion reserve due to coronary microvascular dysfunction. T1 mapping is sensitive to myocardial water content of both intra- and extracellular in origin, but the effect of intravascular compartment changes on T1 has never been assessed previously. We aimed to assess the effect of adenosine-induced vasodilatation on native (pre-contrast) $\mathrm{T} 1$ values in patients with severe aortic stenosis (AS) before and after aortic valve replacement (AVR).

\section{Methods}

42 subjects (26 patients with severe AS without obstructive coronary artery disease and 16 controls) underwent cardiovascular magnetic resonance at 3T for native T1-mapping (ShMOLLI), first-pass perfusion (myocardial perfusion reserve index-MPRI) at rest and

Table 1 Baseline clinical characteristics of severe AS patients and normal controls

\begin{tabular}{|c|c|c|c|}
\hline & Severe Aortic Stenosis $(\mathrm{n}=26)$ & Normal controls $(n=16)$ & $P$ value \\
\hline Age (years) & $67.8 \pm 9$ & $63.3 \pm 3.4$ & 0.06 \\
\hline Male, n (\%) & $19(73)$ & $8(53)$ & 0.16 \\
\hline Body mass index $(\mathrm{kg} / \mathrm{m} 2)$ & $27.8 \pm 4.5$ & $27.0 \pm 3.8$ & 0.38 \\
\hline Systolic blood pressure $(\mathrm{mmHg})$ & $134.4 \pm 18.1$ & $131.0 \pm 11.0$ & 0.51 \\
\hline Diastolic blood pressure $(\mathrm{mmHg})$ & $74.4 \pm 9.4$ & $76.5 \pm 10.2$ & 0.51 \\
\hline Heart rate (bpm) & $66.1 \pm 9.4$ & $64.3 \pm 10.5$ & 0.58 \\
\hline Peak AV gradient (mmHg) & $83.1 \pm 14.6$ & - & \\
\hline \multicolumn{4}{|l|}{ CMR findings } \\
\hline Aortic valve area $(\mathrm{cm} 2)$ & $0.82 \pm 0.02$ & $4.04 \pm 0.75$ & $<0.001$ \\
\hline LV end-diastolic volume (ml) & $143.2 \pm 44.4$ & $133.7 \pm 33.1$ & 0.47 \\
\hline LV ejection fraction (\%) & $74.5 \pm 5.8$ & $68.8 \pm 6.4$ & 0.005 \\
\hline LV mass index $(\mathrm{g} / \mathrm{m} 2)$ & $96.0 \pm 31.2$ & $55.8 \pm 13.9$ & $<0.001$ \\
\hline Presence of LGE, n (\%) & $21(81)$ & 0 & $<0.001$ \\
\hline Myocardial T1 (ms) & $1196 \pm 47$ & $1168 \pm 27$ & 0.037 \\
\hline
\end{tabular}

Values are mean \pm SD or percentages. CMR indicates cardiovascular magnetic resonance; LGE, late gadolinium enhancement; LV, left ventricular. 


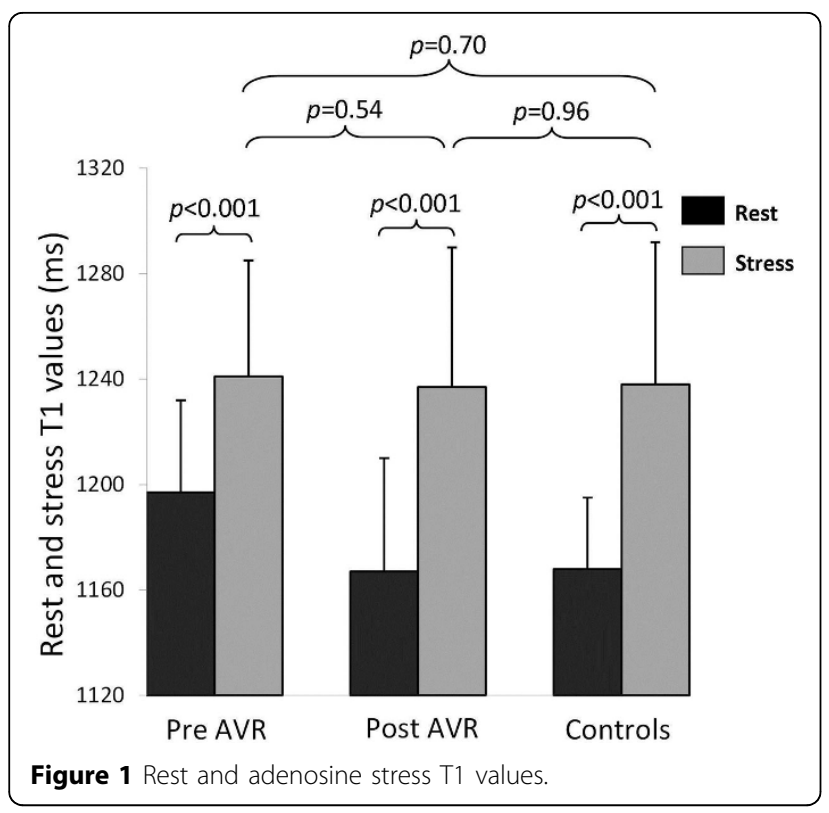

during adenosine stress, and late gadolinium enhancement (LGE).

\section{Results}

AS patients had increased resting myocardial T1 (1196 $\pm 47 \mathrm{~ms}$ vs. $1168 \pm 27 \mathrm{~ms}, \mathrm{p}=0.037)$, reduced MPRI $(0.92$ \pm 0.31 vs. $1.74 \pm 0.32, p<0.001)$, and increased left ventricular mass index (LVMI) and LGE volume compared to controls (Table 1). T1 values had positive correlation with LVMI, LGE volume but an inverse correlation with aortic valve area and MPRI. During adenosine stress, the maximal T1 in AS was similar to controls $(1240 \pm 51 \mathrm{~ms}$ vs. $1238 \pm 54 \mathrm{~ms}, \mathrm{p}=0.88$ ), possibly reflecting a similar level of maximal coronary vasodilatation in both groups (Figure 1). Interestingly, the T1 response to stress was blunted in AS ( $\Delta \mathrm{T} 13.7 \pm 2.7 \%$ vs. $6.0 \pm 4.2 \%$ in controls, $\mathrm{p}=0.013)$. Seven months after AVR $(\mathrm{n}=16)$ myocardial $\mathrm{T} 1$ and response to adenosine stress recovered towards normal.

\section{Conclusions}

Our findings suggest that intravascular compartment is a significant contributor to myocardial T1 relaxation time. Vasodilator stress T1 mapping may be a potential technique to assess coronary reserve without the need for contrast administration. Performing T1 mapping soon after vasodilator stress may affect ECV measurements given that hyperemia alone substantially alters $\mathrm{T} 1$ values.

\section{Funding}

N/A.

\section{Authors' details}

${ }^{1}$ Division of Cardivascular Medicine, Radcliffe Department of Medicine, Oxford Centre for Clinical Magnetic Resonance Research, Oxford, UK. ${ }^{2} 1$ st Department of Cardiology, AHEPA Hospital, Aristotle University of Thessaloniki, Thessaloniki, 54636, Greece.

Published: 3 February 2015

doi:10.1186/1532-429X-17-S1-O28

Cite this article as: Mahmod et al:: Severe aortic stenosis has blunted myocardial $\mathrm{T} 1$ relaxation response to vasodilator stress: a cardiac magnetic resonance adenosine stress test study. Journal of Cardiovascular Magnetic Resonance 2015 17(Suppl 1):O28.
Submit your next manuscript to BioMed Central and take full advantage of:

- Convenient online submission

- Thorough peer review

- No space constraints or color figure charges

- Immediate publication on acceptance

- Inclusion in PubMed, CAS, Scopus and Google Scholar

- Research which is freely available for redistribution

Submit your manuscript at www.biomedcentral.com/submit
C Biomed Central 